\title{
LHD Diagnostics Toward Steady-State Operation
}

Shigeru Sudo, Byron J. Peterson, Member, IEEE, Kazuo Kawahata, Yoshio Nagayama, K. Narihara, Yasuji Hamada, K. Toi, Katsumi Ida, Harukazu Iguchi, Kuninori Sato, S. Morita, Tetsuo Ozaki, Akimitsu Nishizawa, Kenji Tanaka,

T. Minami, Ichihiro Yamada, S. Mutoh, Masahiko Emoto, Hideya Nakanishi, M. Goto, Satoshi Ohdachi, Tokihiko Tokuzawa, Shigeru Inagaki, Takeshi Ido, M. Yoshinuma, Satoru Sakakibara, S. Masuzaki,

Tomohiro Morisaki, Mamoru Shoji, M. Osakabe, and Naoko Ashikawa

Invited Paper

\begin{abstract}
The large helical device (LHD) is the world largest helical system having all superconducting coils. After completion of LHD in 1998, six experimental campaigns have been carried out successfully. The maximum stored energy, central electron temperature, and volume averaged beta value are $1.16 \mathrm{MJ}, 10$ $\mathrm{keV}$, and $3.2 \%$, respectively. The confinement time of the LHD plasma appears to be equivalent to that of tokamaks. One of the most important missions for LHD is to prove steady-state operation, which is also significant to international thermonuclear experimental reactor (ITER) and to future fusion reactors. LHD is quite appropriate for this purpose based upon the beneficial feature of a helical system, that is, no necessity of the plasma current. So far, the plasma discharge duration was achieved up to $150 \mathrm{~s}$. The plasma density was kept constant by feedback control of gas puffing with real time information of the line density. The issue for demonstrating steady-state operation is whether divertor function to control particle and heat flux is effective enough. Relevant to this, LHD diagnostics should be consistent with the following:
\end{abstract}

1) continuous operation of main diagnostics during long-pulse operation for feedback control and physics understanding;

2) measurement of fraction of $\mathrm{H}$, $\mathrm{He}$, and impurities in the plasma;

3) heat removal and measure against possible damage or surface erosion of diagnostic components inside of the vacuum chamber;

4) data acquisition system for handling real time data display and a huge amount of data.

Although there are already some achievements on the above subjects, there remain still several issues to be resolved.

On the other hand, the long-pulse operation of the plasma gives benefits to the diagnostics. For example, the polarizing angle of ECE emission can be changed during the discharge, and the intensity dependence on the polarizing angle has been obtained. The spatial scanning of the neutral particle analyzer and the spectrometer can supply the spatial profiles of the fast neutral particle flux and the specific impurity lines.

In this paper, the present status of these issues and future plans are described.

Index Terms-Fusion reactors, helical system, plasma measurements, stellarators.

Manuscript received July 19, 2003; revised September 11, 2003.

The authors are with the National Institute for Fusion Science, Toki 509-5292,

Japan (e-mail: sudo@LHD.nifs.ac.jp).

Digital Object Identifier 10.1109/TPS.2004.823896

\section{INTRODUCTION}

$\mathbf{I}$ $\mathrm{N}$ RECENT years, magnetic plasma confinement experiments for fusion energy have begun to progress beyond short pulse physics experiments to machines designed to test the feasibility for steady-state plasma confinement, which is essential for reactor operation [1]-[3]. Concomitant with the design and operation of such experiments is the need to develop the measurement capabilities to diagnose such long duration plasmas. In addition, the special challenges posed by a fusion reactor environment demand modifications and new techniques for measuring the parameters needed for reactor operation and plasma characterization [4].

Among the handful of devices, which are currently pursuing the limits of long-pulse, high performance plasmas [1], [2], the large helical device (LHD) is unique in its use of superconducting helical coils (one pair) and vertical field coils (three pairs) in a heliotron configuration $(1 / \mathrm{m}=2 / 10)$ to provide, in steady state, the entire confining magnetic field (up to $2.9 \mathrm{~T}$ ) [3]. This externally formed confining field precludes the need for the driven plasma current which is essential in a tokamak and which limits the density by leading to discharge terminating current disruptions in those types of devices. Therefore LHD is inherently suited to steady-state operation and the development of steady-state diagnostics.

LHD is one of the largest operating magnetic plasma confinement devices with a major radius which can be varied from 3.42 to $4.1 \mathrm{~m}$, a minor radius which averages $60 \mathrm{~cm}$ and a plasma volume on the order of $30 \mathrm{~m}^{3}$ [3]. LHD is well equipped with a variety of plasma heating methods: neutral beam injection (NBI) - 3 beam lines, $150-180 \mathrm{keV}$ negative ion, $\sim 10 \mathrm{MW}$ total; ion cyclotron resonance heating (ICRH) -6 antenna, $\sim 2.7$ MW total; electron cyclotron resonance heating (ECRH) 84 and $168 \mathrm{GHz}, \sim 2.1 \mathrm{MW}$ total. The flexible combination of these heating sources has enabled several notable achievements in LHD: peak electron and ion temperatures exceeding $10 \mathrm{keV}$ [5] and $7 \mathrm{keV} \mathrm{[6],} \mathrm{respectively,} \mathrm{the} \mathrm{formation} \mathrm{of} \mathrm{an} \mathrm{electron} \mathrm{internal}$ transport barrier [7], averaged beta of $\sim 3.2 \%$ and a stored energy of 1.16 MJ [8] and a long-pulse duration of $120 \mathrm{~s}$ [9] which has recently been extended to $150 \mathrm{~s}$. In addition line-averaged electron densities up to $1.6 \times 10^{20} \mathrm{~m}^{-3}$ have been achieved [8] and confinement time scaling which rivals ELMy H-mode tokamaks has been demonstrated [10]. 
The LHD experiment has a five-fold objective, which includes the following:

1) to realize high $\mathrm{n} \tau_{\mathrm{E}} \mathrm{T}$ plasmas and to study the transport physics relevant to fusion plasmas;

2) to demonstrate high $\beta$ stable plasmas $(\langle\beta\rangle \geq 5 \%)$ and to study the related physics;

3) to study energetic particle behaviors in order to simulate $\alpha$ particles in fusion plasmas;

4) to increase the physics understanding of toroidal plasmas by an approach that is complementary with the other helical systems and tokamaks;

5) to develop the physics and technology for steady-state operation and control using a divertor.

In this paper, diagnostic developments related to steady-state plasma experiments on LHD are described. In Section II, the description of diagnostics is divided into four categories: Primary diagnostics for physics studies and their suitability for long-pulse experiments, diagnostics needed specifically for steady-state operation, diagnostics which have been enhanced through long-pulse experiments and development of data acquisition systems for steady-state experiments. In Section III, recent long-pulse experiments on LHD and their diagnosis are described. Finally, in Section IV, unresolved issues related to the steady-state experiments, particularly in a reactor environment are discussed and the article is summarized.

\section{LHD DIAGNOSTICS}

LHD has an extensive set of diagnostics for studying the physics of plasma confinement [11], [44] as shown in Table I. The arrangements of diagnostics and heating systems are shown in Fig. 1.

\section{A. Primary Diagnostics and Suitability for Steady State}

The primary diagnostics on LHD can be classified into five categories: 1) electron density diagnostics; 2) electron temperature diagnostics; 3 ) ion temperature diagnostics; 4) impurity radiation diagnostics; and 5) others. In general, the diagnostics on LHD are working routinely, also during long-pulse experiments. The primary limitation on diagnostics in terms of steady-state operation is the memory size of the data acquisition system. This issue is discussed further is Section II-D. In this section, we will briefly introduce each system and remark on any performance issues related to steady-state operation.

1) Electron Density Diagnostics: On LHD various diagnostics for measuring the electron density exist. A two-color millimeter wave (MMW) interferometer is used to monitor the lineaveraged density [12]. A 13-channel far infrared (FIR) interferometer provides density profile measurements [13]. A multichord $\mathrm{CO}_{2}$ laser imaging interferometer is also under development for detailed profile measurements of density and density fluctuations [14], [45]. In addition reflectometer [15] and polarimeter [16] diagnostics are under development. For edge density measurements Thomson scattering [17] can be used, and also a lithium beam probe has recently begun operation [18].

2) Electron Temperature Diagnostics: Electron temperature measurements include a high spatial resolution YAG laser Thomson scattering system [19] with flexible repetition
TABLE I

LHD Plasma Diagnostics

\begin{tabular}{|c|c|c|}
\hline DIAGNOSTIC & MEASUREMENT & BRIEF DESCRIPTION \\
\hline $\begin{array}{l}\text { Magnetic Probes } \\
\mu W \text { Interferometer }\end{array}$ & $\begin{array}{c}\mathrm{I}_{\mathrm{p}}, \mathrm{W}_{\mathrm{p}}, \text { Plasma Position } \\
n_{\mathrm{e}} \mathrm{L}\end{array}$ & $\begin{array}{l}\text { Rogowski, Mirnov, Flux Loops } \\
2 \mathrm{~mm} / 1 \mathrm{~mm} \text { wave, single channel }\end{array}$ \\
\hline $\begin{array}{l}\text { FIR Laser } \\
\text { Interferometer }\end{array}$ & $n_{e} L(r)$ & $119 \mu \mathrm{m}-\mathrm{CH}_{3} \mathrm{OH}$ laser, 13 chords \\
\hline $\begin{array}{l}\mu \mathrm{W} \text { Reflectometer } \\
\text { Polarimeter }\end{array}$ & NBI Interlock, $\mathrm{n}_{\mathrm{e}}$ Fluct. & $\begin{array}{c}33,35,39,60,65 \mathrm{GHz} \\
10.6 \text { um CO} \text { laser }\end{array}$ \\
\hline Thomson Scattering & $T_{e}(r), n_{e}(r)$ & 130 spatial points, $20 \mathrm{~ms}, 2.5 \mathrm{~J}$ \\
\hline TV Thomson & $T_{e}(r), n_{e}(r)$ & $\begin{array}{c}\text { YAG laser } \\
\text { high spatial resolution }\end{array}$ \\
\hline $\begin{array}{l}\text { ECE (GPC, } \\
\text { radiometer, } \\
\text { Michelson) }\end{array}$ & $\mathrm{T}_{\mathrm{e}}(\mathrm{r}, \mathrm{z})$ 2-D Imaging & $\begin{array}{l}14 \mathrm{ch} \\
32 \mathrm{ch}\end{array}$ \\
\hline X-ray & $\mathrm{T}_{\mathrm{e}}, \mathrm{Im}$ & $20 \mathrm{chs}$ \\
\hline Soft X-r & $T_{e}$, Shafr & Soft X \\
\hline TOF-F & $T_{i}$, Energy Spectra $f(E)$ & Time-of-flight method, radial scan \\
\hline CXS & $T_{i}(r), V_{p}(r)$ & Radial p \\
\hline X-ray Crysta & $T_{i}(r)$ & $0.1-4 \mathrm{~nm}, \lambda$ \\
\hline $\begin{array}{l}\text { Neutron Diagnostics } \\
\text { Bolometers }\end{array}$ & $\begin{array}{c}\text { Neutron flux, } T_{i} \\
P_{\text {rad }}(r) \\
P_{\text {rad }}(r) \text { 2-D Imaging } \\
\text { AXUV Diodes }\end{array}$ & $\begin{array}{l}\text { NE-213 detectors, } 3 \mathrm{He} \text { counters } \\
\text { metal film type } 56 \text { channels } \\
\text { 3 Imaging Bolometer Cameras } \\
\text { 1,2-D tomography } 75 \text { channels }\end{array}$ \\
\hline VUV Spectro & Impurities, $T_{i}$ & $1-200 \mathrm{~nm}, \lambda / \Delta \lambda: 10^{4}$ \\
\hline Visible Spectroscopy & $\begin{array}{l}\mathrm{n}_{0}(\mathrm{H}), \mathrm{Z}_{\text {eff }} \\
\text { Impurity line }\end{array}$ & $\begin{array}{c}200-700 \mathrm{~nm}, \lambda / \Delta \lambda: 5 \times 10^{4} \\
\text { nine } 20 \mathrm{~cm} \text { monochromators, etc. }\end{array}$ \\
\hline Real ti & Visible, $\mathrm{H}_{\alpha}, \mathrm{C}_{\mathrm{l}}$ & tangential core \& divertor views \\
\hline $\begin{array}{l}\text { Soft X-ray Diode Array } \\
\text { Soft X-ray Camera } \\
\mu W / F I R \text { Scattering }\end{array}$ & $\begin{array}{l}\text { MHD Oscillations } \\
\text { MHD Oscillations } \\
\text { Micro-instabilities }\end{array}$ & $\begin{array}{l}\text { silicon surface-barrier diodes } \\
\text { high speed imaging } \\
1 \mathrm{~mm} / 195 \mu \mathrm{m} \text { multichannel }\end{array}$ \\
\hline $\begin{array}{l}\text { Heavy Ion Beam Probe } \\
\text { Langmuir Probes }\end{array}$ & $\begin{array}{l}\Phi p, \Phi p \text { Fluctuation } \\
\mathrm{T}_{\mathrm{e}}, \mathrm{n}_{\mathrm{e}}, \Phi p\end{array}$ & $\begin{array}{l}\mathrm{Au}, 6 \mathrm{MeV}, 100 \mu \mathrm{A} \text { (developing) } \\
\text { Fast scanning and fixed probes }\end{array}$ \\
\hline $\begin{array}{l}\text { Divertor Spectroscopy } \\
\text { Li Beam Probe }\end{array}$ & Recycling, Particle Flux & \\
\hline $\begin{array}{l}\text { High-energy Particle } \\
\text { Diagnostics }\end{array}$ & High-energy Particles & $\begin{array}{c}\text { Silicon Detector Array, Diamond } \\
\text { Detectors, TOF-FNA }\end{array}$ \\
\hline Diagnostic Pellet & Particle Transport & TESPEL/ *TECPEL, C, Li \\
\hline Visible/Infrared TV & Plasma position, PWI & TV system \\
\hline Fast Ion Gauge & Neutral Gas Pressure & \\
\hline $\begin{array}{l}\text { Magnetic Surface } \\
\text { Mapping }\end{array}$ & Magnetic Surface & $\begin{array}{l}\text { Electron Beam, } \\
\text { Fluorescent Mesh and Rod }\end{array}$ \\
\hline
\end{tabular}

(from microseconds to hundreds of milliseconds) as shown in Fig. 2. The calibration for the density is not yet completed, so the density profile should be taken only as a reference. In the figure, the short and long intervals are $300 \mu \mathrm{s}$ and $100 \mathrm{~ms}$, respectively. These numbers can be changed according to the purpose. In addition to this, a TV Thomson scattering system has been introduced recently with the goal of the high spatial resolution. Also, three types of electron cyclotron emission (ECE) diagnostics have been installed, grating polychromator, radiometer and Michelson [20]. The ECE is proven as a reliable diagnostic for the temporal development of the electron temperature profile. The absolute electron temperature agrees well with the Thomson scattering data in case of medium density. When the electron density increases to $n_{e}>0.6 n_{\text {cutoff }}$, then the ECE temperature starts to decrease due to the diffraction of the ray. This is due to the highly elliptical and twisted shape of the LHD plasma resulting in a curved plasma surface in the viewing field of the ECE antenna. Therefore, the refraction of the ECE ray is not negligible if the electron density is more than half of the cut off density. The refraction causes the diffusion of the ECE ray so that the detected ECE intensity is reduced. Soft X-ray diagnostics include a pulse height analyzer system [21] and a soft X-ray charge-coupled device (CCD) imaging system [22]. The electron temperature measured by the Soft 


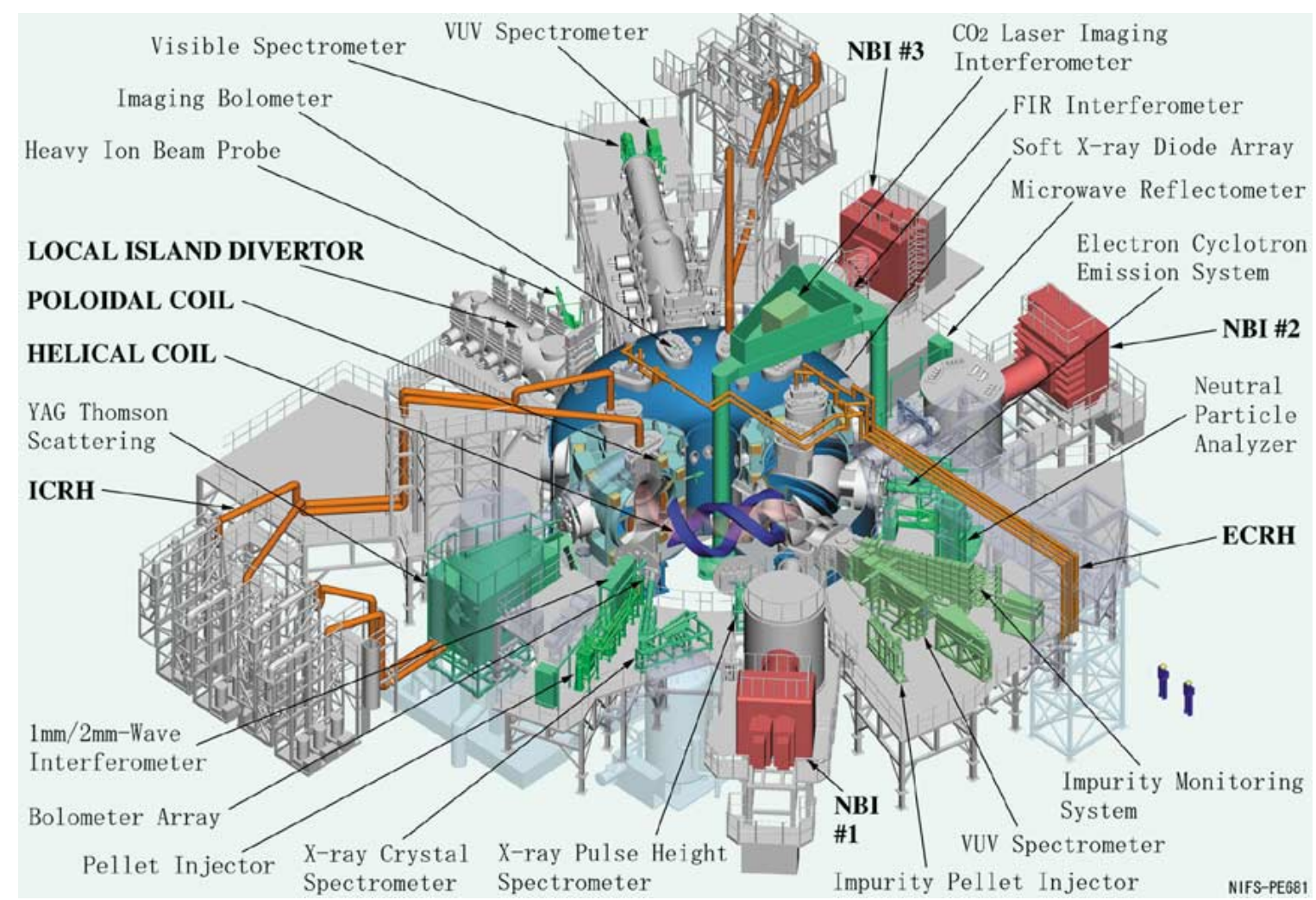

Fig. 1. Arrangement of diagnostics and heating systems on LHD.

X-ray diagnostics agrees well also with the data measured by the Thomson scattering system.

3) Ion Temperature Diagnostics: Ion temperature is measured on LHD using a crystal spectrometer for peak temperature (and also toroidal rotation velocity) [23], a charge exchange recombination spectroscopy (CXRS) system for profile information [24], and neutral particle analyzers for energy spectra [25], [26], [46], [47]. The highest central ion temperature is obtained from the Doppler broadening of Ar XVII measured by the crystal spectrometer.

4) Impurity Radiation Diagnostics: Impurity radiation from the LHD plasma is monitored with various arrays of spectrometers and bolometers. A 2-m soft X-ray duo-multichannel spectrometer (SOXMOS) (0.5-34 nm) [27], a 3-m VUV spectrometer using a CCD detector to provide a vertical profile of the spectra [28] and a 50-cm UV-visible Czerny-Turner type spectrometer (200-550 nm) [29] have been installed. An impurity monitoring station consisting of nine $20-\mathrm{cm}$ normal incidence monochromators $(30-180 \mathrm{~nm})$, a $2.2-\mathrm{m}$ grazing incidence monochromator $(10-120 \mathrm{~nm})$, a $20-\mathrm{cm}$ normal incidence polychromator $(30-550 \mathrm{~nm})$, a flat field extreme UV polychromator (1-10 nm, planned), $\mathrm{H}_{\alpha}$ and $\mathrm{He}_{\mathrm{I}}$ visible monitors, total radiation monitor (using a secondary electron multiplier), and a soft X-ray monitor [30]. The X-ray pulse height analyzer is also used to measure heavy impurity emission [21]. Bolometers of three different types (resistive-56 ch, absolute extreme UV photodiode (AXUVD) - $75 \mathrm{ch}$ and infrared (IR) imaging - three cameras) are used to measure the total radiated power distribution from the plasma [31]. Resistive bolometers suffer from an uncompensated thermal drift of the signal which is difficult to correct in the case of long-pulse experiments. One possible solution to this problem would be to have two sets of bolometers with shutters which would be alternately closed for zeroing the amplifiers. Tracer encapsulated solid pellets are used to investigate impurity transport [32].

5) Other Diagnostics: For measuring the electric field the CXRS system is used [24] and a heavy ion beam probe is under development. MHD fluctuations are studied using arrays of soft $\mathrm{X}$-ray detectors, mirnov coils and the ECE diagnostics [20]. The soft X-ray detectors include PIN photodiode arrays [33] and a high-speed soft X-ray imaging camera [34]. In addition magnetic probes are used to measure the stored energy, plasma current and the plasma position. Magnetic probes and other diagnostics using integrator circuits are not suitable for continuous operation beyond $\sim 10 \mathrm{~s}$ due to saturation of the integrated signal. One possible countermeasure to this problem is the use of multiple integrator circuits, which are multiplexed to produce a continuous measurement. Such a system is currently under testing at NIFS. Another possible solution uses a rotating coil probe and has been tested on the TRIAM device [35]. Langmuir probes are installed in several locations in the divertor strike plates and also a fast scanning probe is used to measure plasma parameters in the divertor leg region [36].

\section{B. Diagnostics for Steady-State Experiments}

Diagnostics which are essential for steady-state operations can be classified as either those for real-time monitoring or those for feed-back control. Real-time monitoring is necessary to insure that certain parameters do not exceed the limits of safe 

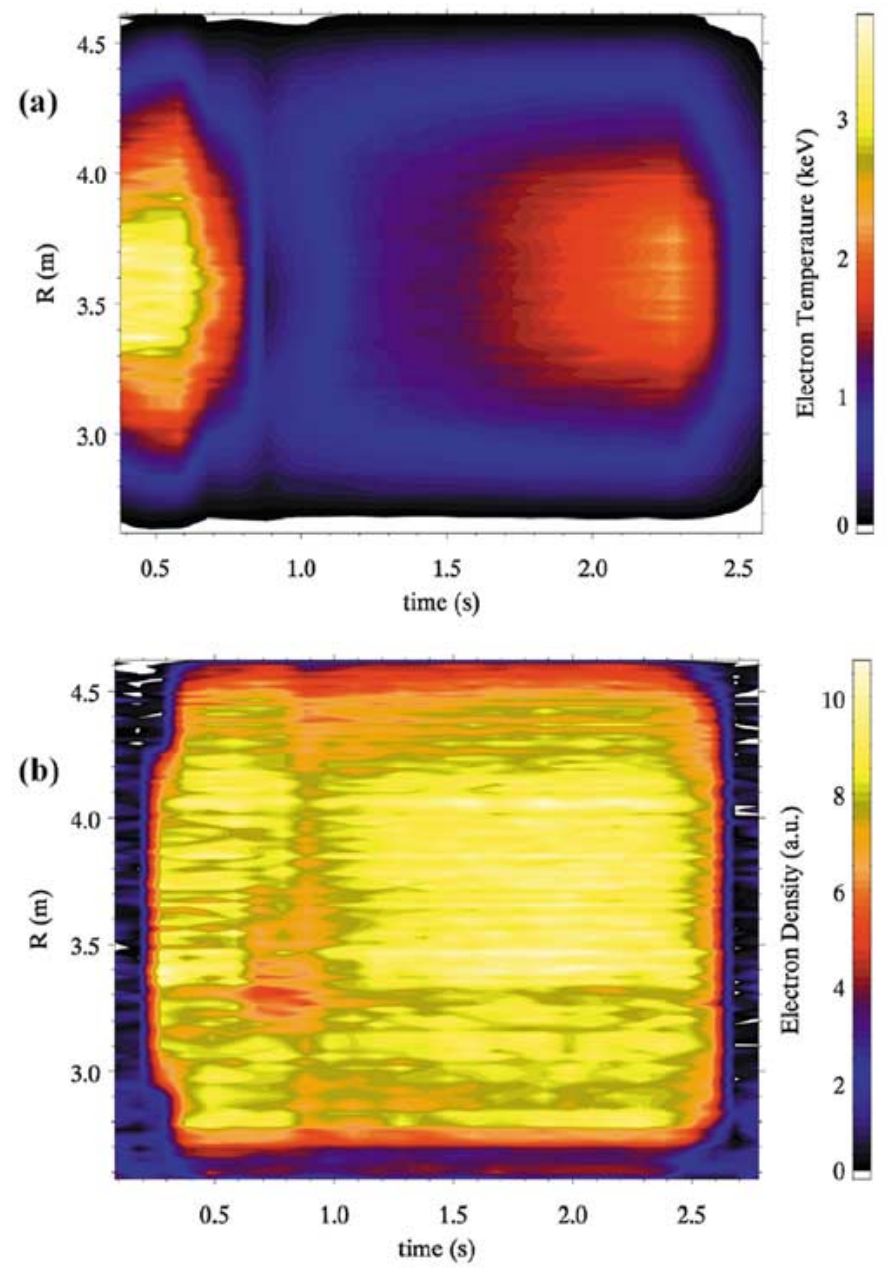

Fig. 2. One example (Shot no. 33621) of (a) electron temperature and (b) density profiles measured by a high spatial resolution YAG laser Thomson scattering system.

operation. Feedback control is necessary when one measured parameter is used to control a device in order to maintain the plasma in a steady-state condition. These diagnostics and their use on LHD are described in the following.

1) Real Time Monitoring: Real-time monitoring and display of various parameters carried out during long-pulse experiments in LHD using the WE7000 system described below in Section II-D. These include core line-averaged electron density from the FIR interferometer, electron temperature at several radii from the ECE radiometer, $\mathrm{H}_{\alpha}, \mathrm{C}_{\mathrm{II}}$, and $\mathrm{O}_{\mathrm{V}}$ signals from the impurity monitoring station, total radiated power from AXUV photodiodes, ion saturation current from divertor Langmuir probes and the temperatures of vacuum window flanges using thermocouples at two different locations. In addition to these, real-time video signals of visible light and of various impurity lines $\left(\mathrm{H}_{\alpha}, \mathrm{C}_{\mathrm{III}}\right.$, and $\left.\mathrm{O}_{\mathrm{II}}\right)$ are available [37]. One example of the CCD camera (3-O) view with $\mathrm{H} \alpha$ filter at the bottom part of vertically elongated cross-section is shown in Fig. 3, and the calculated magnetic field line trace is also shown in Fig. 3. The characteristic structure agrees well. Real time video is also used to monitor ICRF antennas and the Local Island Divertor head for arcing and hot spots. Also, IR thermography measurements monitor the temperature of

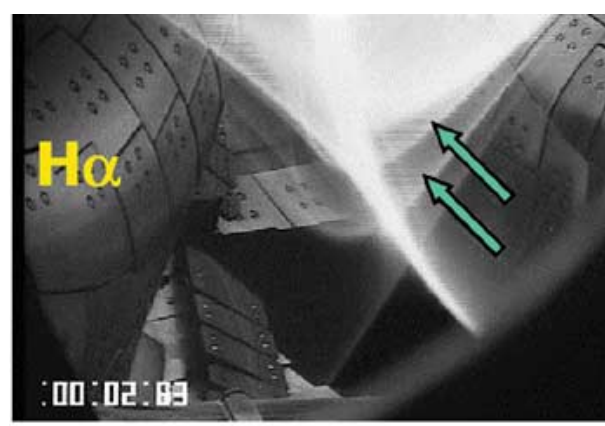

a)

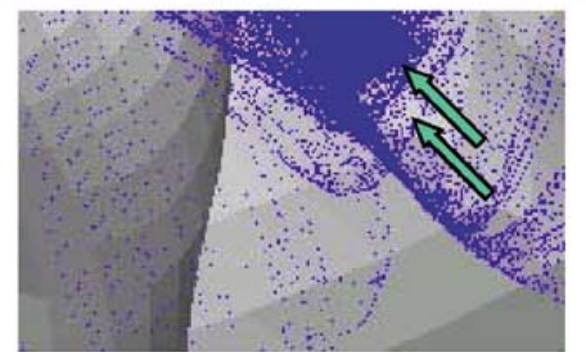

b)

Fig. 3. (a) $\mathrm{CCD}$ camera (3-O) view with $\mathrm{H} \alpha$ filter at the bottom part of vertically elongated cross-section. (b) Calculated magnetic field line trace.

the graphite armor tiles of the neutral beam injection (NBI) beam dumps [38]. Finally, for machine operation purposes numerous thermocouples are installed throughout LHD [36] and are sampled continuously and displayed at a 1-Hz sampling rate along with measurements of the vacuum tank and cryostat pressures.

2) Feed-Back Control: Various signals are used for feedback control of various devices to insure steady-state conditions and device protection. Line averaged density signals from the FIR interferometer are used to control the gas puff to maintain a constant density during long-pulse discharges. Plasma current signals from the Rogowski coils can be used to adjust the coil currents to control the plasma current. This is done by calculating the necessary coil currents to change the plasma current from the measured value to the target value and then adjusting the coil currents accordingly in real time with a 100-ms control period [39]. These plasma current signals, in addition to measurements of the super conducting coil currents, can be used to keep the coil currents constant or to keep the total magnetic flux through the coils constant. A reflectometer is used to monitor the density as an interlock signal for the NBI, to turn off the beams in case of a premature termination of the plasma. The super-conducting coils are protected by emergency shutdown which can be triggered by various conditions: excessive coil balance voltage; loss of vacuum in coil cryostat; loss of power supply; strong earthquake; etc.

\section{Benefits to Diagnostics From Steady-State Operation}

Several diagnostics can benefit from the long time scales of steady-state experiments. The VUV spectrometer can be slowly rotated vertically $(0.022-0.044 \mathrm{deg} / \mathrm{s})$ during a steady-state discharge to measure the spatial profile of the spectra [27]. As shown in Fig. 4, radial distributions of spectral line intensities have been obtained during a long-pulse discharge. Taking advantage of a stationary state of the plasma, the localization property depending on ionization degree was clearly observed with 


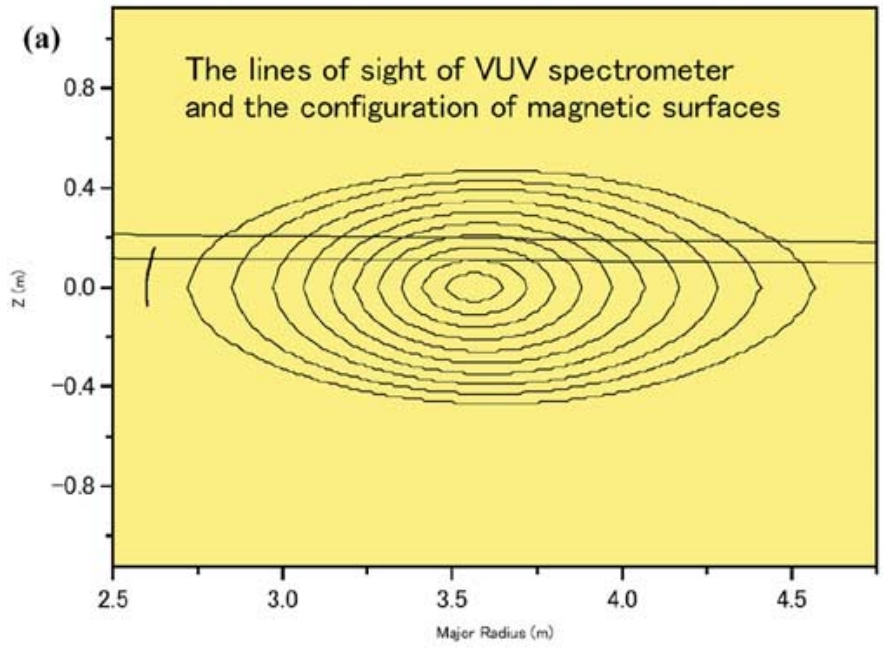

(b)

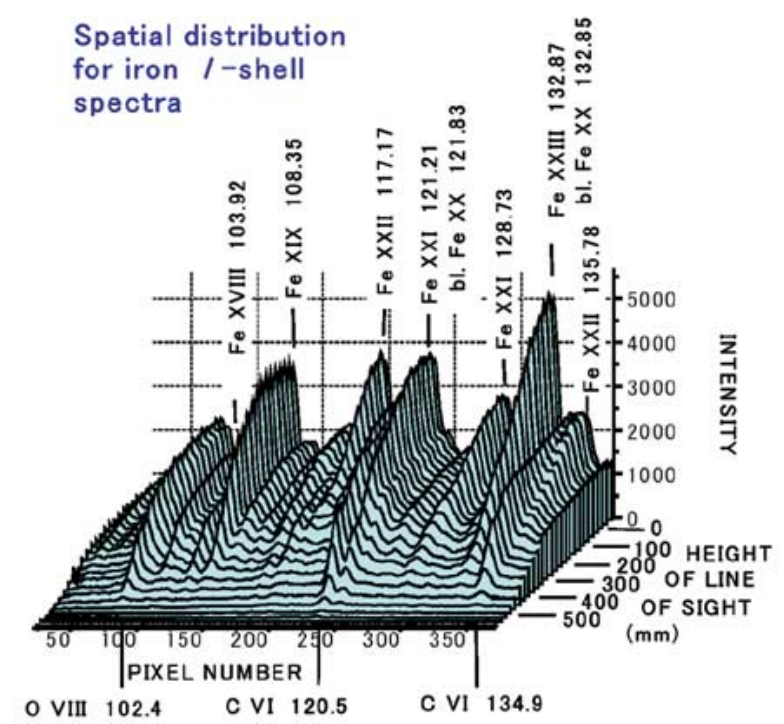

Fig. 4. VUV spectrometer can be (a) rotated vertically during a steady-state discharge to measure the (b) spatial profile of the spectra.

chord scanning. The 3-m VUV spectrometer is also designed to be scanned toroidally providing a two-dimensional diagnostic [28]. The polarizer for the ECE diagnostics [20] can be rotated during the experiment to give a real-time measurement of the polarization. The viewing angle of the NPA [25] can be scanned to provide a measure of the loss cone. Many diagnostics can benefit from increased integration times to improve photon statistics or spatial resolution, at the cost of time resolution during discharges which are held in a steady state for a long period of time. Examples of these diagnostics are TV Thomson scattering, $\mathrm{X}$-ray CCD camera [22] and imaging bolometers [30].

\section{Data Acquisition}

The data acquisition system for the LHD experiment uses two systems, the standard one for data acquisition for post shot analysis and one for real-time display. The real-time display is handled by a Yokogawa WE7000 system ${ }^{1}$, which can sample at 20 $\mathrm{kHz}$ and display up to 32 channels in real-time. The standard
TABLE II

SuMMARY OF LONG-PULSE ACHIEVEMENTS IN LHD

\begin{tabular}{|c|c|c|c|c|}
\hline $\begin{array}{c}\text { Heating } \\
\text { source }\end{array}$ & $\begin{array}{c}\text { Input } \\
\text { power } \\
(\mathrm{kW})\end{array}$ & $\begin{array}{c}\text { Duration } \\
\text { (s) }\end{array}$ & $\begin{array}{l}\text { Density } \\
\left(10^{18} / \mathrm{m}^{3}\right)\end{array}$ & $\begin{array}{c}\text { Plasma } \\
\text { temperature } \\
(\mathrm{keV})\end{array}$ \\
\hline $\mathrm{ECH}$ & 50 & 120 & 0.3 & $\mathrm{~T}_{\mathrm{e}} \sim 0.65$ \\
\hline NBI & 600 & 80 & 16 & $\mathrm{~T}_{\mathrm{e}} \sim 1.5$ \\
\hline NBI & 100 & 110 & 10 & $\mathrm{~T}_{\mathrm{e}} \sim 0.35$ \\
\hline $\mathrm{ICH}$ & 350 & 120 & 8 & $\mathrm{~T}_{\mathrm{e}} \sim \mathrm{T}_{\mathrm{i}} \sim 1.3$ \\
\hline $\mathrm{ICH}$ & 520 & 150 & 6 & $\mathrm{~T}_{\mathrm{e}} \sim \mathrm{T}_{\mathrm{i}} \sim 2$ \\
\hline
\end{tabular}

LHD data acquisition system is based on a massively parallel CAMAC (30 crates, 2000 diagnostic channels) /PC (30 sets) /RAID (50 GB/PC) acquisition hardware, hierarchical double/mirrored storage media (RAID-2 TB, MO jukebox-1.2 TB, DVD-ROM changer-3.2 TB), and a cluster of data retrieval clients (50), all connected by a gigabit-based network structure. The raw data are stored in an object-oriented database which handles over 700 MB (100 MB compressed)/shot with approximately 150 shots per experiment day with one shot every 3 min for standard short pulse experiments (less than $10 \mathrm{~s}$ ). For long-pulse experiments typically the CAMAC sampling time is lengthened to extend the acquisition period to match the pulselength, however, for those diagnostics that require it (for example diagnostics used to investigate fluctuations), event-driven triggering is available for short acquisition periods with high sampling speeds. Dual alternating systems with iterative operation (2-3 min) are envisioned to allow indefinite continuous data acquisition and storage at intermediate sampling rates $(1-5 \mathrm{kHz})$.

A new real-time data acquisition system, which would replace the current CAMAC system, and is based on compact platform component interconnect (PCI) technology ${ }^{2}$ is currently under prototype testing at NIFS in cooperation with National Instruments Corporation. The objective is to reach $1 \mathrm{MS} / \mathrm{s} / \mathrm{ch}$ with $100 \mathrm{MB} / \mathrm{s}$ continuous data transfer. Currently, achieved parameters are $2.5 \mathrm{MS} / \mathrm{s} / \mathrm{ch}$ and $84-\mathrm{MB} / \mathrm{s}$ transfer rate with improvements expected as systems are gradually upgraded [40].

\section{LHD STEADY-STATE EXPERIMENTS}

Long-pulse experiments have progressed steadily [3] since LHD's first experimental campaign to reach the parameters listed in Table II. Some notable achievements have been the control of heavy impurities through the introduction of a graphite helical divertor [41], and steady-state fueling by means of continuous repetitive $(11 \mathrm{~Hz})$ injection of cryogenic Hydrogen pellets [42], [48]. The vacuum vessel walls and the helical divertor are actively cooled with flowing water with the capability to remove $0.3 \mathrm{MW} / \mathrm{m}_{2}$ of power at the divertor in steady state. This design would allow $3 \mathrm{MW}$ of input power assuming all was conducted to the divertor. Therefore, the development of heating power supplies is being pursued with the long term goal of $3 \mathrm{MW}$ of ICH heating for one hour. The short term goal for the next experimental campaign is to

\footnotetext{
${ }^{2}$ National Instruments Inc. http://www.ni.com.
} 

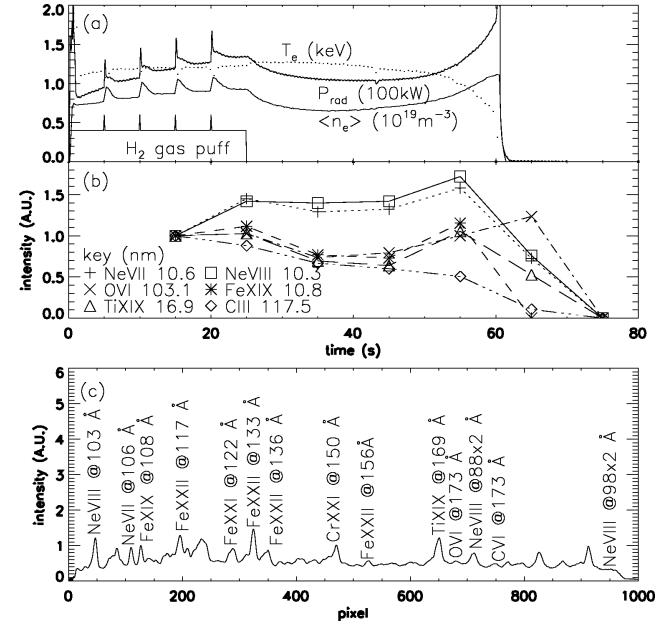

Fig. 5. Long-pulse discharge in LHD heated with NBI and terminated by radiative collapse showing: (a) time evolution of major parameters: line-averaged electron density from FIR interferometer, electron temperature from ECE and total radiated power from AXUVD with hydrogen gas puffing; (b) time evolution of selected spectral lines from the VUV spectrometer; and (c) impurity radiation spectra in the $10 \ddot{y} 20 \mathrm{~nm}$ region from the VUV spectrometer just prior to the radiative collapse.

TABLE III

UNRESOLVED ISSUES FOR STEADY STATE FUSION REACTOR DiagNostics

\begin{tabular}{cc}
\hline \hline Issue & Counter Measure \\
\hline Heat removal & Water cooling plasma facing components (PFCs) \\
Erosion & Modular construction of PFC for easy replacement \\
Magnetic & Multiplexing of integrator circuits \\
measurements & \\
Real-time & Event triggering, C-PCI based system \\
monitoring & \\
Nuclear & Shielding, cooling of affected parts \\
heating & Collective Thomson Scattering, TESPEL CXS \\
measurement & \\
\hline
\end{tabular}

perform a 5-min ECH discharge with $100 \mathrm{~kW}$ of input power. In Fig. 5, the desorption effect during an NBI long-pulse experiment is shown with emphasis on the time evolution of the neon radiation [9]. In this plasma, the working gas was hydrogen, but neon plasma experiments had been done in the previous days.

\section{DISCUSSION CONCLUSION}

In this paper, we have described the diagnostics in LHD in terms of their suitability for and benefits from long-pulse experiments. However, several topics related to diagnostics for a fusion reactor have not been mentioned and are listed in Table III; these include heat removal, shielding from neutrons, nuclear heating, and activation.

In the case of heat removal for diagnostics in LHD, most diagnostics are mounted on the large port flanges outside of the vacuum vessel. Since they are relatively far from the plasma the steady-state heat load from radiation is quite low $\left(<1 \mathrm{~kW} / \mathrm{m}^{2}\right)$ and can be handled by the connection to the vacuum vessel. As a precautionary measure, diagnostics that are located inside the vacuum vessel and have components that might outgas at high temperature are water-cooled (e.g., SX diode arrays and AXUVD arrays). In a reactor environment, however, much more attention should be paid to heat removal as the heat loads would be much higher.

Another important issue is the coating of vacuum windows by dust and other deposited materials. Analysis of dust collected from LHD after the third campaign (in March of 2001) showed very small amounts of $\mathrm{Fe}-\mathrm{C}$ composite dust spread throughout the machine [43]. In terms of coating of windows, shutters are used to prevent this from happening during glow discharge cleaning and wall conditioning. However, the large number of discharges during one campaign (up to 10000) has lead to some loss of transmission in some windows. For instance the Thomson scattering system has a large vacuum window which is covered on the vacuum side by another window $(65 \times 40 \times 12 \mathrm{~mm})$ which is replaced annually. The reduction in transmission on this window is typically less than $10 \%$. Slight reductions in transmission have been observed in other windows in the visible range, but this effect is harder to estimate for the UV range.

Also in a reactor, the consequences of the high-energy neutrons must be considered. This would include neutron shielding of sensitive diagnostics, remote handling for maintenance of activated components, nuclear heating of diagnostics, etc. Since in LHD we will not use tritium fuel and the expected neutron fluxes from planned D-D experiments are much lower than expected in a reactor, this is not an important issue for LHD.

Other important and challenging issues for reactors are the diagnosis of fast neutron fluxes of the transport of fast alpha products. However, these reactor-related issues are difficult to address in a nonreactor environment.

\section{REFERENCES}

[1] M. Sakamoto et al., "Global particle balance and wall recycling properties of long duration discharges on TRIAM-1M," in Fusion Energy 2002: Proc. 19th Int. Conf., Lyon, France, 2002, IAEA-CN-94/EX/P4-07, pp. 1-5.

[2] C. Laviron and Tore Supra Team, "Diagnostics and steady-state high power operation," Rev. Sci. Instrum., vol. 74, pp. 1770-1772, Mar. 2003.

[3] N. Noda et al., "Experimental studies toward long-pulse steady-state operation in LHD," Nucl. Fusion, vol. 41, pp. 779-790, June 2001.

[4] P. Stott et al., Advanced Diagnostics for Magnetic and Inertial Fusion. Norwell, MA: Klewer Academic, 2002.

[5] S. Kubo et al., "Achievement of $10 \mathrm{keV}$ central electron temperatures by ECH in LHD," J. Plasma Fusion Res., vol. 78, pp. 99-100, Feb. 2002.

[6] Y. Takeiri et al., "Achievement of a high ion temperature with $\mathrm{Ne}$ - and Ar-seeded discharges by high power NBI heating in LHD," in Proc. 30th EPS Conf. Control Fusion and Plasma Physics, St. Petersbug, Russia, 2003, 2.171, pp. 1-4.

[7] — "Formation of electron internal transport barrier and achievement of high ion temperature in large helical device," Phys. Plasmas, vol. 10, pp. 1788-1795, May 2003.

[8] A. Komori et al., "Recent results from the large helical device," Plasma Phys. Contr. Fusion, vol. 45, pp. 671-686, May 2003.

[9] Y. Nakamura et al., "Plasma performance and impurity behavior in longpulse discharges on LHD," Nucl. Fusion, vol. 43, pp. 219-227, Apr. 2003.

[10] H. Yamada et al., "Energy confinement and thermal transport characteristics of net current free plasmas in the large helical device," Nucl. Fusion, vol. 41, pp. 901-908, July 2001.

[11] S. Sudo et al., "Overview of large helical device diagnostics," Rev. Sci. Instrum., vol. 72, pp. 483-491, Jan. 2001.

[12] K. Kawahata, K. Tanaka, Y. Ito, A. Ejiri, and R. J. Wylde, "A twocolor millimeter-wave interferometer for the measurement of line integral electron density on large helical device," Rev. Sci. Instrum., vol. 70, pp. 695-698, Jan. 1999. 
[13] K. Kawahata, K. Tanaka, Y. Ito, A. Ejiri, and S. Okajima, "Far infrared interferometer system on the large helical device," Rev. Sci. Instrum., vol. 70, pp. 707-709, Jan. 1999.

[14] K. Tanaka et al., "Phase contrast imaging interferometer for edge density fluctuation measurements on LHD," Rev. Sci. Instrum., vol. 74, pp. 1633-1637, Mar. 2003

[15] T. Tokuzawa et al., "X-mode pulsed radar reflectometer for density fluctuation measurements on LHD," Rev. Sci. Instrum., vol. 74, pp. 1506-1509, Mar. 2003

[16] T. Akiyama et al., "Faraday rotation densitometry for large helical device," Rev. Sci. Instrum., vol. 72, pp. 1073-1076, Jan. 2001.

[17] I. Yamada et al., "Raman calibration of the LHD YAG Thomson scattering for electron-density measurements," Rev. Sci. Instrum., vol. 74 pp. 1675-1678, Mar. 2003

[18] T. Morisaki et al., "Lithium beam probe for edge density profile measurements on the large helical device," Rev. Sci. Instrum., vol. 74, pp. 1865-1868, Mar. 2003

[19] K. Narihara et al., "Design and performance of the Thomson scattering diagnostic on LHD," Rev. Sci. Instrum., vol. 72, pp. 1122-1125, Jan. 2001.

[20] Y. Nagayama et al., "Electron cyclotron emission diagnostics on the large helical device," Rev. Sci. Instrum., vol. 70, pp. 1021-1024, Jan. 1999.

[21] S. Muto et al., "First measurement of hard X-ray spectrum emitted from high-energy electrons in electron cyclotron heated plasma of LHD," Rev. Sci. Instrum., vol. 74, pp. 1993-1996, Mar. 2003.

[22] Y. Liang et al., "Photon-counting CCD detector as a tool of X-ray imaging," Rev. Sci. Instrum., vol. 72, pp. 717-720, Jan. 2001

[23] S. Morita and M. Goto, "X-ray crystal spectrometer with a charge-coupled-device detector for ion temperature measurements in the large helical device," Rev. Sci. Instrum., vol. 74, pp. 2375-2387, Apr. 2003.

[24] K. Ida, S. Kado, and Y. Liang, "Measurements of poloidal rotation velocity using charge exchange spectroscopy in a large helical device," Rev. Sci. Instrum., vol. 71, pp. 2360-2366, June 2000.

[25] T. Ozaki, S. Murakami, and P. Goncharov et al., "Spatial resolved high-energy particle diagnostic system using time-of-flight neutral particle analyzer in large helical device," Rev. Sci. Instrum., vol. 74, pp. 1878-1882, Mar. 2003

[26] J. F. Lyon et al., "Spatially resolved measurements of energetic neutral particle distributions in the large helical device," Rev. Sci. Instrum., vol. 74, pp. 1873-1877, Mar. 2003

[27] T. Kobuchi et al., "Observation of charge-exchangespectra on $\mathrm{C}^{6+}+\mathrm{H}$ in low-energy collision," J. Plasma Fus. Res., vol. 5, pp. 167-170, 2002.

[28] S. Morita and M. Goto, "Space-resolved VUV spectroscopy using the 3 $\mathrm{m}$ normal incidence spectrometer with back-illuminated CCD detector in the LHD," Rev. Sci. Instrum., vol. 74, pp. 2036-2039, Mar. 2003.

[29] M. Goto et al., "Determination of the hydrogen and helium ion densities in the initial and final stages of a plasma in the large helical device by optical spectroscopy," Phys. Plasmas, vol. 10, pp. 1402-1410, May 2003.

[30] S. Morita and M. Goto, "First results from impurity monitor station of LHD," NIFS, 1998-1999 Annu. Rep., 1999.

[31] B. J. Peterson et al., "Bolometer diagnostics for one- and two-dimensional measurements of radiated power on the large helical device," Plasma Phys. Contr. Fusion, vol. 45, pp. 1167-1182, July 2003.

[32] S. Sudo et al., "Particle transport diagnostics on CHS and LHD with tracer-encapsulated solid pellet injection," Plasma Phys. Contr. Fusion, vol. 44, pp. 129-135, Jan. 2002.

[33] S. Ohdachi, K. Toi, M. Takechi, and S. Yamamoto, "Soft X-ray detector array system on the large helical device," Rev. Sci. Instrum., vol. 72, pp. 727-730, Jan. 2001

[34] S. Ohdachi et al., "High-speed tangentially viewing soft X-ray camera to study magnetohydrodynamic fluctuations in toroidally confined plasmas (invited)," Rev. Sci. Instrum., vol. 74, pp. 2136-2143, Mar. 2003.

[35] J. Fujita et al., "A rotating coil probe for the magnetic field measurement on a long-pulsed tokamak," Rev. Sci. Instrum., vol. 70, pp. 445-448, Jan. 1999.

[36] S. Masuzaki et al., "The divertor plasma characteristics in the large helical device," Nucl. Fusion, vol. 42, pp. 750-758, June 2002.

[37] M. Shoji, K. Yamazaki, and S. Yamaguchi, "Development of the real-time image data acquisition system for observing the plasma dynamic behavior of LHD long-pulse discharges," J. Plasma Fusion Res., vol. 3, pp. 440-443, 2000.

[38] M. Osakabe et al., "In situ calibration of neutral beam port-through power and estimation of neutral beam deposition on LHD," Rev. Sci. Instrum., vol. 72, pp. 590-593, Jan. 2001.
[39] K. Nishimura et al., "Real-time plasma current control experiment," $J$. Plasma Fusion Res., vol. 5, pp. 265-269, 2002.

[40] H. Nakanishi, M. Kojima, and M. Ohsuna et al., "Steady-state data acquisition method for LHD diagnostics," Fusion Eng. Des., vol. 66-68, pp. 827-832, Sept. 2003.

[41] Y. Nakamura et al., "Particle balance in NBI heated long-pulse discharges on LHD," J. Nucl. Mater., vol. 290-293, pp. 1040-1044, Mar. 2001.

[42] H. Yamada et al., "Repetitive fueling pellet injection in large helical device," Fusion Eng. Des., vol. 69, pp. 11-14, Sept. 2003.

[43] J. P. Sharpe et al., "Characterization of dust collected from ASDEXupgrade and LHD," J. Nucl. Mater, vol. 313-316, pp. 455-459, Mar. 2003.

[44] S. Sudo et al., "Recent diagnostic developments on LHD," Plasma Phys. Contr. Fusion, vol. 45, pp. 1127-1142, July 2003.

[45] T. Akiyama, " $\mathrm{CO}_{2}$ laser imaging interferometer for high spatial resolution electron density profile measurements on LHD," Rev. Sci. Instrum. vol. 74, pp. 1638-1641, Mar. 2003.

[46] T. Saida et al., "Charge exchange fast neutral measurement with natural diamond detectors in neon plasma on LHD," Rev. Sci. Instrum., vol. 74 pp. 1883-1886, Mar. 2003.

[47] M. Osakabe et al., "Development and energy calibration of Si-FNA for LHD fast ion measurement," Rev. Sci. Instrum., vol. 72, pp. 788-791, Jan. 2001

[48] R. Sakamoto et al., "Repetitive pellet fuelling on LHD," in Proc. 30th EPS Conf. Controlled Fusion and Plasma Physics, vol. 27A, Europhysics Conf. Abstr., St. Petersburg, Russia, July 2003, P-3.12, pp. $1-4$.

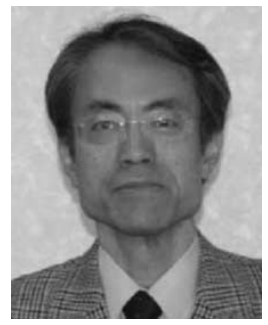

Shigeru Sudo received the Dr.Sci. degree in plasma physics from Tokyo University, Tokyo, Japan, in 1977.

After postdoctoral work with the Institute of Plasma Physics, Nagoya University, Nagoya, Japan, he joined the Institute of Plasma Physics, Max-Planck-Institute (IPP-MPI), Garching, Germany, in 1977, and was a Junior Staff Member from 1979 to 1980 . In 1980, he joined Kyoto University, Kyoto, Japan, and he engaged in the Thomson scattering diagnostics and pellet injection for the Heliotron E project. Since 1992, he has been with the Large Helical Device Project at the National Institute for Fusion Science, Toki, Japan, where he is currently developing the tracer-encapsulated pellet diagnostics method.

Dr. Sudo is the Deputy Director-General of the National Institute for Fusion Science.

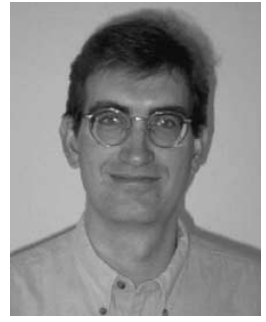

Byron J. Peterson (M'83) received the Ph.D. degree in electrical engineering from the University of Wisconsin-Madison, in 1994, for his work on the measurement of currents and flows in the interchangeable module stellarator.

After graduation, he continued his research on the Heliotron-E device as a Postdoctoral Fellow for one year. In 1995, he joined the National Institute for Fusion Science, Toki, Japan, as an Assistant Professor responsible for the development of bolometer diagnostics for the large helical device (LHD). Since the beginning of LHD experiments in 1998, he has focused on the development of imaging bolometers and the study of radiative phenomena in LHD.

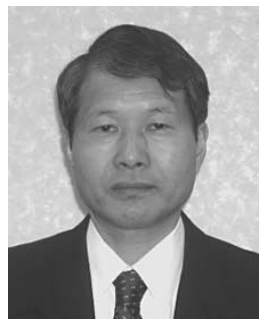

Kazuo Kawahata received the $\mathrm{Ph}$. D. degree in physics from Nagoya University, Nagoya, Japan in 1976.

After receiving the $\mathrm{Ph}$. D degree, he joined the Research Staff at the Institute of Plasma Physics, Nagoya University, Nagoya, Japan, specializing in millimeter/submillimeter wave diagnostics for magnetic fusion plasmas. Since 1989, he has been with the Large Helical Device (LHD) Project at the National Institute for Fusion Science, Toki, Japan. His current research interests include the experimental physics of the LHD and the development of millimeter/submillimeter wave diagnostics needed to investigate plasma characteristics in fusion experiments. 


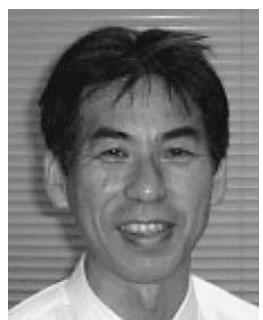

Yoshio Nagayama received the Ph.D. degree in the plasma physics from University of Tokyo, Tokyo, Japan, in 1979.

After working for the plasma control in tokamak and reverse field pinch in University of Tokyo, he engaged in the imaging diagnostics or tomography and investigated the MHD instabilities and the reconnection in TFTR tokamak at the Princeton Plasma Physics Laboratory, Princton, NJ. In 1994, he joined the Large Helical Device Project at the National Institute for Fusion Science, Toki, Japan, where he is currently investigating plasma physics for nuclear fusion, especially the electron cyclotron emission diagnostics and the data analysis system.

K. Narihara, photograph and biography not available at the time of publication.

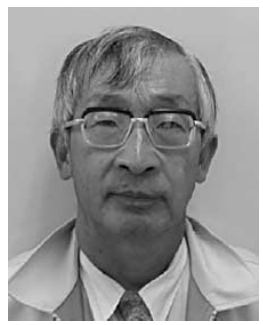

Yasuji Hamada received the Ph.D. degree from the University of Tokyo, Tokyo, Japan, in 1971. His studies included the research of toroidal turbulent heating in a figure-8 stellarater.

After graduation, he joined the Institute for Plasma Physics, Nagoya University, Nagoya, Japan. He also worked on the dc-Octupole (internal ring machine) at General Atomics, San Diego, CA, and on the current drive in tokamak plasma at the Physics Department, Kyoto University, Kyoto, Japan. He then rejoined the Institute for Plasma Physics, Nagoya University, later started to work on the heavy ion beam probe (HIBP) measurement on Japanese Institute of Plasma Physics (JIPP) T-2U tokamak, JFT-2M tokamak in JAERI, and on the design and construction on 6-MeV HIBP for large helical devices.

K. Toi, photograph and biography not available at the time of publication.

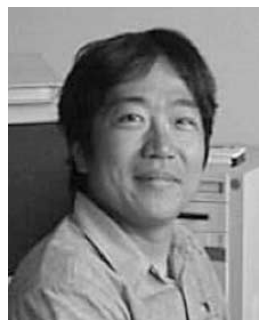

Katsumi Ida received the Ph.D. degree in physics from the University of Tokyo, Tokyo, Japan, in 1986.

His research interests include the mechanism of reduction of turbulent transport by the radial electric field shear and/or magnetic field shear in toroidal plasmas using a charge of charge exchange spectroscopic and motional stark effect spectroscopy. He has also been working on both in tokamak plasmas (JIPPTIIU, JFT-2M, JET, and JT60U) and helical plasmas (CHS, Heliotron-E, Wendelstein 7AS and large helical devices) and trying to get comprehensive understandings on turbulent transport in toroidal plasmas.

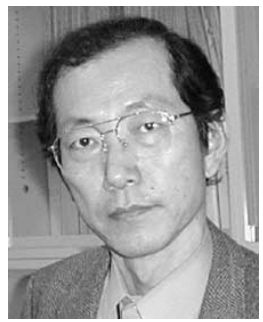

Harukazu Iguchi received the Ph.D. degree in plasma physics from Nagoya University, Nagoya, Japan in 1979.

From 1977 to 1986, he was engaged in research on plasma confinement in a Nagoya bumpy torus (NBT) at the Institute of Plasma Physics, Nagoya University. In 1987, he was with the RF heating group, JET Joint Undertaking, Abingdon, U.K. Since 1988, he has been working on diagnostics for the low-aspectratio helical device CHS at the National Institute for Fusion Science, Toki, Japan, especially on Thomson scattering and beam probe diagnostics such as a heavy ion beam probe (HIBP) and a lithium neutral beam probe (LiBP).

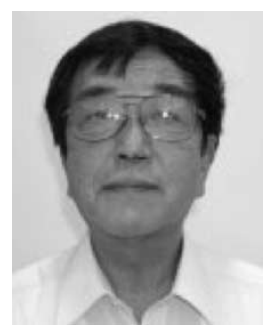

Kuninori Sato received the Ph.D. degree in population inversion at recombining plasma from Nagoya University, Nagoya, Japan, in 1977.

After postdoctoral work with the Institute of Plasma Physics, Nagoya University, he joined the Research Staff of Institute of Plasma Physics, Nagoya University, in 1979, where his research included the diagnostics, especially vacuum ultraviolet (VUV) spectroscopic measurements in the Japanese Institute of Plasma Physics (JIPP)-TIIU tokamak. In 1989, he joined the Large Helical Device Project at the National Institute for Fusion Science, Toki, Japan, where he is currently investigating VUV and EUV measurement and analysis using several spectorometers.
S. Morita, photograph and biography not available at the time of publication.

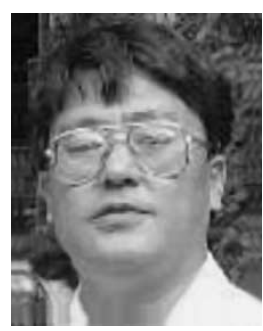

Tetsuo Ozaki received the Ph.D. degree in particle beam fusion from Osaka University, Osaka, Japan, in 1985.

After postdoctoral work with the Institute of Laser Engineering, Osaka University, he moved to Institute for Plasma Physics, Nagoya University, Nagoya, Japan, in 1986. There, he engaged in diagnostics, especially visible spectroscopic measurements including the charge exchange spectroscopic measurement and the neutral particle measurement in Japanese Institute of Plasma Physics (JIPP-TIIU) and compact helical system (CHS). In 1989, he joined the Large Helical Device Project at the National Institute for Fusion Science, Toki, Japan, where he is currently investigating high-energy particle measurement and analysis using several neutral particle analyzers.

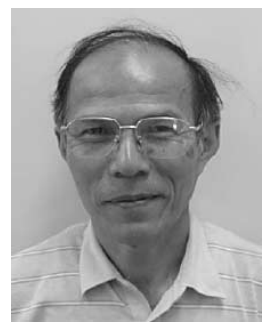

Akimitsu Nishizawa received the Ph.D. degree in physics from Nagoya University, Nagoya, Japan, in 1972.

After graduation, he was with the Institute for Plasma Physics, Nagoya University, where he was engaged in diagnostics, especially the heavy ion beam probe in Japanese Institute of Plasma Physics (JIPP)-TIIU. In 1989, he joined the Large Helical Device Project at the National Institute for Fusion Science, Toki, Japan, where he is currently developing HIBP using a 3-MV tandem accelerator.

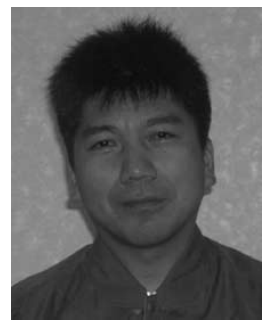

Kenji Tanaka received the $\mathrm{Ph} . \mathrm{D}$. degree from Kyusyu University, Fukuoka, Japan, in 1995. His studies focused on microturbulence using laser phase contrast interferometer on Heliotorn E.

$\mathrm{He}$ has been with the diagnostic division of $\mathrm{Na}-$ tional Institute for Fusion Science, Toki, Japan, since 1993. He developed and constructed far infrared interferometer, polarimeter and reflectometer to measure electron density, internal magnetic field and electron density fluctuation in compact helical systems and large helical devices. His research interests include particle transports and role of microturbulence on it by using far infrared and infrared interferometer.

T. Minami, photograph and biography not available at the time of publication. 


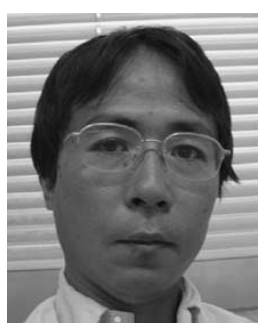

Ichihiro Yamada received the Dr.Sci degree from Nagoya University, Nagoya, Japan, in 1990. His studies included experimental studies of atomic processes in fusion plasmas.

In 1991, he developed the compact helical system and large helical device (LHD) YAG Thomson scattering systems while at the National Institute for Fusion Science, Toki, Japan. His research interests include the diagnostics of electron temperature and density profiles of LHD plasmas with the YAG Thomson scattering, and the development of a new TV Thomson scattering on LHD

S. Mutoh, photograph and biography not available at the time of publication.

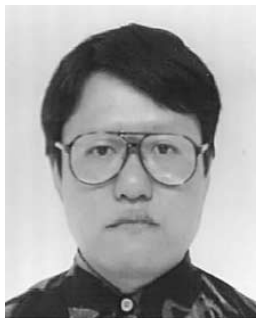

Masahiko Emoto received the Ph.D. degree in nuclear fusion from the Graduate University for Advanced Stduies, Kanagawa, Japan, in 2003.

He had been a Computer Engineer with Nihon Sun Microsystems K.K., Tokyo, Japan, for six years, and then joined the National Institute for Fusion Science, Toki, Japan, in 1998. He then joined the Large Helical Device Project, and has been engaging in the data management system.

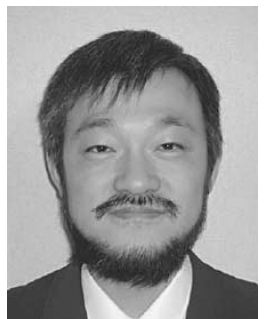

Hideya Nakanishi received the Ph.D. degree in engineering from the Department of Nuclear Engineering, University of Tokyo, Tokyo, Japan, in 1995

Since 1995, he has engaged in the R\&D for the data acquisition and diagnostic control computing systems in the Large Helical Device Project at the National Institute for Fusion Science, Toki, Japan. His recent research interests are to establish the technologies of the ultra-wideband $(\sim 1 \mathrm{Gbps})$ real-time data acquisition and storage system for steady-state fusion experiments.

M. Goto, photograph and biography not available at the time of publication.

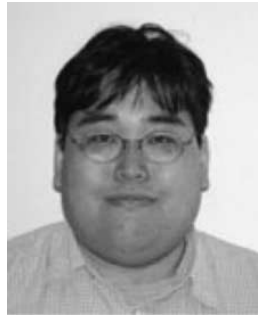

Satoshi Odachi received the M.S. degree in plasma physics from the Department of Physics, Waseda University, Tokyo, Japan, in 1989 and the Dr.Eng. degree from the Department of Energy Engineering and Science, Nagoya University, Nagoya, Japan, 2003

He has been with the National Institute for Fusion Science (NIFS), Toki, Japan, since 1994, where he has been interested in the fluctuations in plasma. His research interests include edge fluctuations using Langmuir probes in JFT-2M (JAERI) and Japanese Institute of Plasma Physics (JIPP) TII-U (NIFS) tokamaks, and the compact helical device (CHS) (NIFS) device. He also studies MHD instabilities by soft $\mathrm{X}$-ray measurement using detector array system on CHS and LHD devices. He is currently developing a tangentially viewing soft X-ray camera fluctuation study fluctuations in collaboration with Institute of Plasma Physics, Juelich, Germany. After experiments in LHD, the camera system was moved to the TEXTOR tokamak. He is now temporally staying in Germany and doing experiments with this camera.

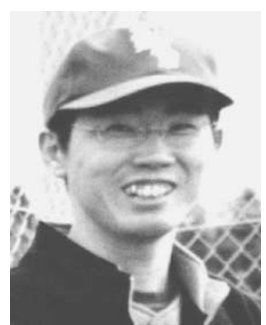

Tokihiko Tokuzawa received the Ph.D. degree from University of Tsukuba, Tsukuba, Japan, in 1998.

In 1998, he joined the Large Helical Device Project at the National Institute for Fusion Science, Gifu, Japan, where he is currently investigating electron density profile and fluctuation measurement by using the electromagnetic wave.
Shigeru Inagaki received the Ph.D. degree in heliac plasma confinement physics from Tohoku University, Sendai, Japan, in 1997.

$\mathrm{He}$ joined the National Institute for Fusion Science, Toki, Japan, in 1997. He engaged in the diagnostics, especially electron cyclotron emission measurements in large helical devices (LHDs). He is currently investigating electron heat transport in LHD plasma.

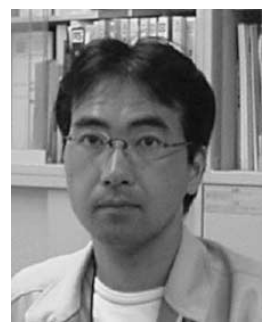

Takeshi Ido received the Ph.D. degree from Nagoya University, Nagoya, Japan, in 2000.

During his graduate work, he participated in th experiments on the JFT-2M tokamak at the Japan Atomic Energy Research Institute, Tokai, where he worked on the development of a heavy ion beam probe (HIBP) for measuring the space potential and density fluctuation. He researched the role of electric field at the formation of the edge transport barrier by using the HIBP. In 2000, he joined the Large Helical Device (LHD) project at the National Institute for Fusion Science, Gifu, Japan. Currently, he is working on the construction on 6-MeV HIBP for LHD.

M. Yoshinuma, photograph and biography not available at the time of publication.

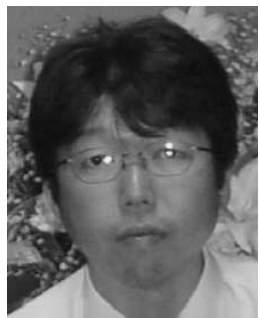

Satoru Sakakibara received the Ph.D degree from the Graduate University for Advanced Studies, Hayama, Japan, in 1995.

That same year, he joined the Large Helical Device Project at the National Institute for Fusion Science, Gifu, Japan. His research interests include the characteristics of MHD equilibrium and stability in helical plasmas using magnetic measurements.

S. Masuzaki, photograph and biography not available at the time of publication.

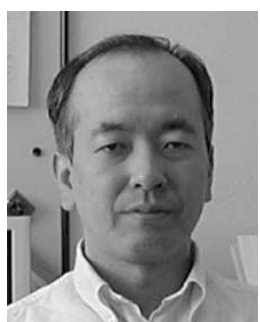

Tomohiro Morisaki received the M.S. and Ph.D. degrees from Kyushu University, Fukuoka, Japan.

Since 1995, he has been with the Large Helical Device Project at the National Institute for Fusion Science, Gifu, Japan. His current research interests include edge plasma physics and active edge control with divertor, together with the edge diagnostics. 


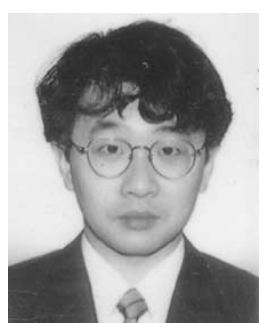

Mamoru Shoji received the Ph.D. degree from the Plasma Research Center, Tsukuba University, Ibaraki, Japan, in 1995.

After postdoctoral work with the Plasma Research Center, he joined the National Institute for Fusion Science, Toki-city, Gifu, Japan, in 1995, where he was with the Plasma Operations Division working in the Large Helical Device Project. He joined in the development of a magnetic surface measurement and a control data processing system for an LHD operation. Since 1998, he has been investigating plasma image data and the analysis of plasma-wall interactions on divertor plates using a neutral particle transport simulation code.

M. Osakabe, photograph and biography not available at the time of publication.

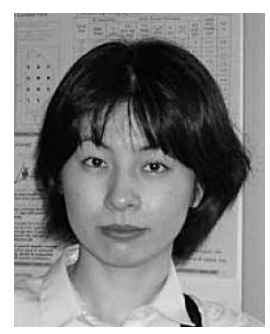

Naoko Ashikawa received the Ph.D. degree for the development of IR imaging bolometers from the Graduate University for Advanced Studies, Gifu, Japan, in 2002.

In 1999, she joined the Large Helical Device (LHD) Project with the National Institute for Fusion Science (NIFS), Gifu, Japan, where she is currently investigating wall conditionings and a plasma radiation structure by IR imaging bolometer. 